\title{
Primary Carcinoid Tumor of the Skull Base: Case Report and Review of the Literature
}

\author{
Mohannad Ibrahim, MD, Mohammad Yousef, MD, Nicholas Bohnen, MD, Avraham Eisbruch, MD, Hemant Parmar, MD \\ From the Department of Radiology (MI, HP); Department of Pathology (MY); Department of Nuclear Medicine (Radiology) (NB); and Division of Radiation Oncology, University \\ of Michigan Health System, Ann Arbor, MI (AE).
}

[Correction added after online publication 29-September-2010: Dates of receipt has been corrected.]

\section{A B S T R A C T}

We present an unusual case of primary intracranial carcinoid tumor of the skull base centered at the level of the foramen jugulare, which was proven with surgical biopsy and later with Somatostatin receptor nuclear medicine scan. We present the salient magnetic resonance imaging features of this rare tumor, describe their characteristic nuclear medicine findings, and briefly review the literature.
Keywords: Skull base, carcinoid, neoplasm, imaging.

Acceptance: Received August 3, 2008, and in revised form August 11, 2008. Accepted for publication August 13, 2008.

\begin{abstract}
Correspondence: Address correspondence to Hemant Parmar, MD, Department of Radiology, University of Michigan, 1500 E Medical Center Drive, Ann Arbor, Ml 48109-0302. E-mails: parurad@hotmail.com and hparmar@ umich.edu.
\end{abstract}

Disclosure: The authors report no conflicts of interest.

J Neuroimaging 2010;20:390-392.

DOI: $10.1111 / j .1552-6569.2008 .00317 . x$

\section{Introduction}

Carcinoid tumors are rare, slow-growing neuroendocrine neoplasms that most commonly arise in the gastrointestinal tract $(62-67 \%)$ or the bronchopulmonary airways $(22-27 \%) .{ }^{1,2}$ Intracranial involvement by a carcinoid tumor is most commonly seen due to hematogeneous metastasis. Primary intracranial carcinoid tumors are rarely mentioned in the literature. ${ }^{3,4} \mathrm{We}$ report a case of a pathologically proven isolated carcinoid tumor of the skull base. Extensive metastatic workup revealed no other primary lesion, indicating this to be the primary site of involvement.

\section{Case Report}

A 29-year-old female presented with progressive multiple cranial nerve palsy including diplopia on rightward gaze, difficulty with swallowing, and hoarseness of voice over the last 6 years. She had complaints of headache, dizziness, and generalized weakness. On examination she had decreased pinprick sensation on the right side of her face with decreased abduction of the right eye, suggestive for right $\mathrm{V}$ and VI cranial nerve palsies, respectively. There was decreased elevation of the right side of her soft palate and right-sided deviation of the tongue on protrusion, suggestive of CN IX and XII palsies, respectively. Her sternocleidomastoid and trapezius muscles had full strength. The patient denied any difficulty breathing, diarrhea, or flushing. Twenty-four-hour urinalysis demonstrated no evidence of elevation of 5-HIAA levels.
Magnetic resonance imaging (MRI) of the skull base demonstrated a large mass lesion centered at the right foramen jugulare extending superiorly into the cerebellomedullary angle cistern (Figs 1A, B) and inferiorly into the right carotid and paravertebral spaces of the upper neck (Fig 1C) and infiltration of the ipsilateral cavernous sinus. Frank osseous invasion of the right petrous temporal bone and right lateral aspect of the clivus was noted. The lesion was predominantly isointense to the gray matter on T1-weighted images (WI) and T2 WI. It showed diffuse moderate homogeneous enhancement following contrast administration. Differential diagnosis based on imaging included glomus jugulotympanicum, schwannoma, meningioma, or metastasis.

Excisional biopsy of the lesion revealed a tan pink, wellcircumscribed soft tissue mass. The tissue had a firm, homogenous yellow-to-pink cut surface. Light microscopy showed islands of monotonous cells with scant, pink granular cytoplasm, round-oval stippled nuclei with small nucleoli, and minimal pleomorphism, with absence of necrosis and mitotic activity (Fig 2A). Further immunohistochemical analysis with neuroendocrine markers stained positive for chromogranin A (Fig 2B) and synaptophysin (2B), and the lesion was diagnosed as carcinoid tumor.

Following the biopsy result, the patient underwent a Somatostatin receptor scintigraphy study using Indium ${ }^{111}$ Pentetreotide [ ${ }^{111}$ In-DTPA-D-Phe-] (Octreoscan), at 24 and 48 hours (Fig 1D). It demonstrated avid radiotracer uptake in the right upper neck and right skull base region, which corresponded to the known lesion at the right skull base. No additional area 
A

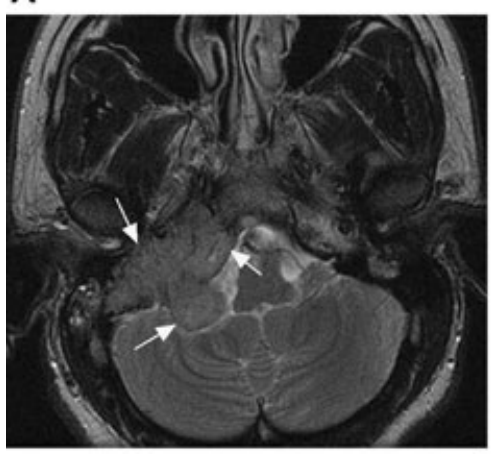

C

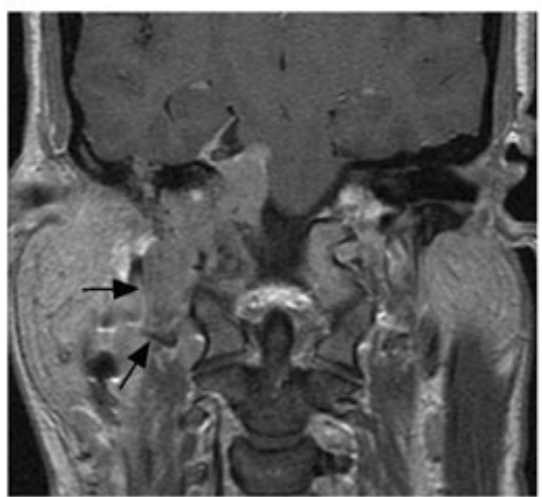

B

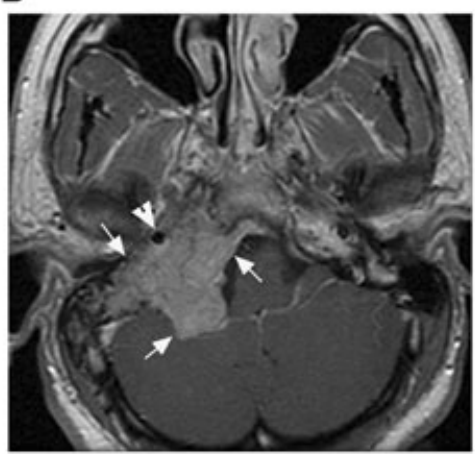

D

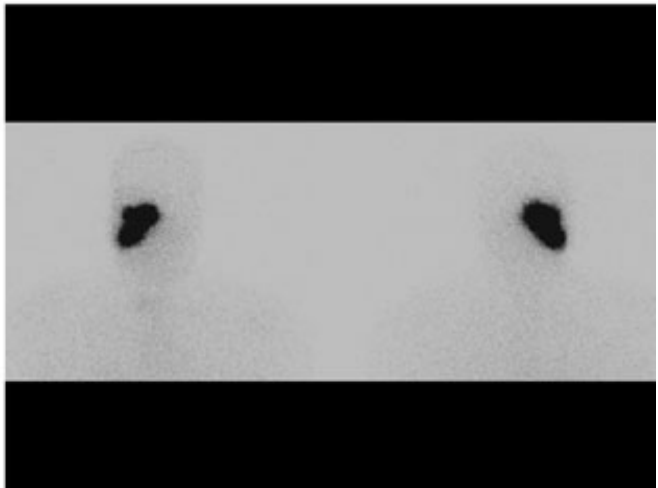

Fig 1. MRI of the brain and skull base demonstrates carcinoid tumor centered the right foramen jugulare extending superiorly into the cerebellomedullary angle cistern (arrows). The lesion is predominantly isointense to the gray matter on T1-weighted images (WI) (not shown) and T2 WI (A). It shows diffuse moderate homogeneous enhancement following contrast administration (B). There is inferior extension into the right carotid space with forward displacement of the right internal carotid artery (arrowhead). There is extension into paravertebral spaces of the upper neck (C) (black arrows) and into the ipsilateral cavernous sinus (not shown). (D) Planar anterior (left) and posterior (right) 48-hour Octreotide images of the head, neck, and chest demonstrate avid radiotracer uptake in the upper right neck and right skull base region.

of abnormal uptake was noted. Extensive imaging of the chest, abdomen, and pelvis showed no evidence of primary neoplasm or additional metastasis. She was treated with monthly somatostatin injections. She denied local radiation treatment. At 1-year follow-up, her symptoms are stable and there was no interval increase in the tumor size.

\section{Discussion}

Carcinoid tumors are relatively uncommon tumors arising from Kulchitsky cells, an enterochromaffin cell located in the crypts of Lieberkuhn of the intestinal lumen. ${ }^{1}$ They do not present clinically until there has been metastatic spread or evidence of carcinoid syndrome. ${ }^{1}$ Carcinoid syndrome is rarely manifested in patients with central nervous system (CNS) lesions. ${ }^{3}$ Deshaies and Huang reported CNS carcinoid mimicking a meningioma, ${ }^{3,4}$ while other primary carcinoid tumors have been reported in the orbit and middle ear. ${ }^{5,6}$ Very few (less than 2\%) patients with carcinoid tumors exhibit CNS metastasis, the commonest primary site being the lungs. ${ }^{7,8}$ Most patients with brain metastasis have either primary lung tumors or metastasis to the lung at the time of brain metastasis. ${ }^{7,8}$ Purely intraparenchymal lesions are found in $58 \%$ of cases while $42 \%$ of the lesions have meningeal involvement. ${ }^{6}$ Carcinoid metastasis to the brain oc- curs after a variable time course with the median interval 16-18 months (range $0-16.3$ years). ${ }^{8}$

Imaging of carcinoid tumor is often challenging, requiring a combination of anatomic and functional techniques. ${ }^{9}$ Imaging techniques include computed tomography (CT), MRI, somatostatin receptor $(\mathrm{SR})$ scintigraphy, and, sometimes, positron emission tomography. Imaging on MRI is nonspecific and with findings similar to more common pathologies like glomus tumor, meningioma, schwannoma, and metastasis. As most carcinoid tumors express type 2 SRs, scintigraphy is widely used as the primary imaging method for diagnosis, staging, and monitoring of carcinoid tumors. ${ }^{10} \mathrm{SR}$ scintigraphy performed using Indium $^{111}$-labeled Octreotide is a sensitive method of localizing radiologically occult carcinoid tumors, with a reported sensitivity of $80 \%-100 \% .{ }^{11,12}$ Whole-body imaging technique may provide valuable information about unsuspected metastatic disease especially when single photon emission CT (SPECT) data are also obtained.

Primary intracranial carcinoid tumors are rare; consequently, once a carcinoid tumor of the brain is suspected, it is imperative to conduct a full body imaging using a combination of anatomic and functional imaging to rule out any additional lesions. 

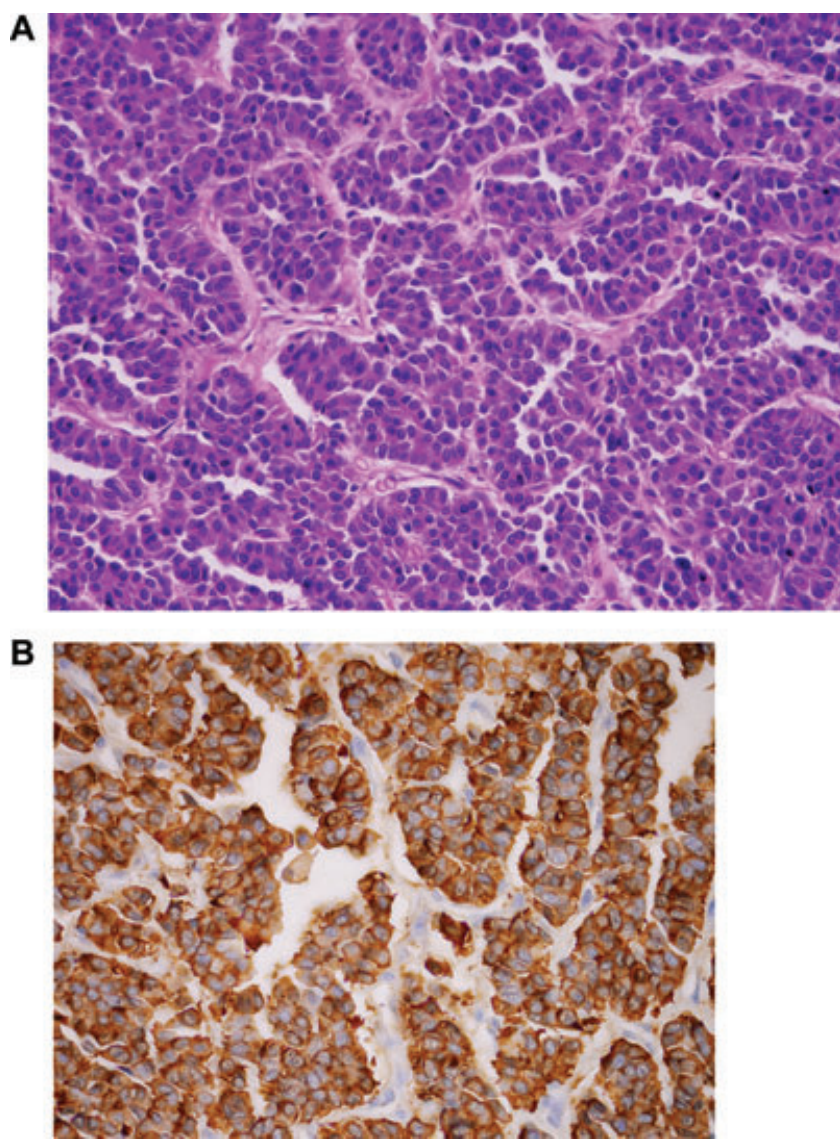

Fig 2. Findings on histopathology. (A) Islands of monotonous cells with scant, pink granular cytoplasm and round-oval stippled nuclei (H\&E, 400×). Chromogranin (B) and synaptophysin immunostain show cytoplasmic positivity of the tumor cells, suggestive for carcinoid tumor.

\section{References}

1. Taal BG, Visser O. Epidemiology of neuroendocrine tumours. Neuroendocrinology 2004;80:3-7.

2. Irvin M M, Lye KD, Kidd M. A 5-decade analysis of 13,715 carcinoid tumors. Cancer 2003;97:934-959.

3. Deshaies EM, Adamo MA, Qian J, et al. A carcinoid tumor mimicking an isolated intracranial meningioma. J Neurosurg 2004;101:858-860.

4. Huang PS, Oz M. Malignant carcinoid tumor metastatic to the dura mater simulating a meningioma. Neurosurgery 1991;29:449452

5. Porter DG, Chakrabarty A, McEvoy A, et al. Intracranial carcinoid without evidence of extracranial disease. Neuropathol Appl Neurobiol 2000;26:298-300.

6. Isidori AM, Kaltsas G, Frajese V. Ocular metastases secondary to carcinoid tumors: the utility of imaging with ${ }^{123} \mathrm{I}-\mathrm{MIBG}$ and ${ }^{111}$ In-DTPA pentetreotide. J Clin Endocrinol Metab 2002;87:16271633.

7. Hlatky R, Suki D, Sawaya R. Carcinoid metastasis to the brain. Cancer 2004;101:2605-2613.

8. Patchell RA, Posner JB. Neurologic complications of carcinoid. Neurology 1986;36:745-749.

9. Kaltsas G, Korbonits M, Heintz E, et al. Comparison of somatostatin analog and MIBG radio-nuclides in the diagnosis and localization of advanced neuroendocrine tumors. J Clin Endocrinol Metab 2001;86:895-902.

10. Reubi JC. Somatostatin and other peptide receptors as tools for tumor diagnosis and treatment. Neuroendocrinology 2004;80:5156.

11. Pfannenberg AC, Eschmann SM, Horger M, et al. Benefit of anatomical-functional image fusion in the diagnostic workup of neuroendocrine neoplasms. Eur J Nucl Med Mol Imaging 2003;30:835-843.

12. Kaltsas G, Rockall A, Papadogias D, et al. Recent advances in radiological and radionuclide imaging and therapy of neuroendocrine tumours. Eur J Endocrinol 2004;151:15-27. 